\title{
Research on Complex Product Parts Matching by using Improved Taguchi Method
}

\author{
Fengque PEI****, Yifei TONG***, Minghai YUAN****, Haojie SONG***** \\ *College of Mechanical and Electrical Engineering, Hohai University, Changzhou, 213000, China, \\ E-mail: FQ_Pei@163.com \\ **AKM Electronics Technology (Suzhou) Company Ltd, Suzhou, 215000, China, E-mail: FQ-Pei@hhu.edu.cn \\ ***School of Mechanical Engineering, Nanjing University of Science \& Technology, Nanjing, 210094, China, \\ E-mail: tyf51129@aliyun.com \\ ****College of Mechanical and Electrical Engineering, Hohai University, Changzhou, 213000, China, \\ E-mail:Ymhai@hhu.edu.cn; \\ *****Nanjing Research Institute of Electronic Engineering, Nanjing, 210007, China, \\ E-mail:15051891899@163.com \\ cross ref http://dx.doi.org/10.5755/j02.mech.28182
}

\section{Introduction}

In 2011, following the concept of "Industry 4.0" proposed by Germany [1], countries around the world came up with targeted policies. Such as, "Digitizing European Industry" in EU [2]." Advanced Manufacturing Partnership and Smart Manufacturing" in US, "Made in China 2025" in China [3-5], "Manufacturing Innovation Strategy3.0" [6] in Korean [7], a boom of intelligent manufacturing has emerged in the world. As the most influential national strategic technology industry in today's world, the equipment manufacturing industry, as a complex product, represents the comprehensive strength of a country. Its healthy and orderly development will bring huge economic and social benefits to a country. In 2010, China proposed a development plan ("Report on accelerating the cultivation of strategic emerging industries") for the complex equipment manufacturing Industry, calling for an overall improvement of the innovation and industrial development capacity of the space industry. Subsequently, high-end CNC machine, aerospace equipment, marine engineering equipment and rail transit equipment were listed as the key development fields in the "Made in China 2025". China will usher in an important period of strategic opportunities for the development of highend equipment manufacturing industry in the next 5-10 years.

The high-end equipment is characterized by complexity. Its assembly is usually discrete with long operation cycle. Manual assembly and customized production are also the challenge. Taking the aircraft assembly line as an example, it mainly includes fuselage, wing, tail, engine, take-off /landing suspension and chassis. For example, the Airbus A320 contains 500,000 to 1.2 million parts in total. During the assembly, the number of rivets exceeds 3,000. Many scholars at home and abroad have been focusing on the assembly process.

The main idea of assembly optimization is to improve the assembly precision by optimizing the assembly tolerance zone. W. Xiao [8] proposes an optimization method of quality point control threshold for complex mechanical product assembly process based on Hybrid Particle Swarm optimization (H-PSO) under multiple loads. By defining the multi-load influencing factors of the key quality control points, the assembly loss/control threshold model is established. In the model, the objective function is built with the minimizing the total cost under the assembly accuracy. L. Chun [9] uses the modelling method combining forward and reverse design to conduct statistical analysis on the distribution of assembly deviation of the front face through Monte Carlo method in the research of assembling electric vehicle, and obtains the optimal tolerance allocation zone. $\mathrm{S}$. Yu [10] proposes a forecasting model of assembly deviation propagation. In the model, the typical deviation characteristics based on the small displacement spin theory and the assembly deviation transfer based on the series assembly relation are established.

While these methods mentioned above have a glaring defect. The tolerance has been decided in the product design period. And the essence of optimizing the tolerance is optimization design process, minimize the overall tolerance. This method is useful for new products or new components, while for the existing assembly process, the optimization is invalid. What's more, the process of enlarged or reduced tolerance is improve machining accuracy. And this method is largely restricted to the accuracy of machine tools. A lot of optimization results are theoretical.

Therefore, some scholars put forward the tolerance matching in view of these disadvantages. The earliest matching is unitized assembly. W w. [11] divided the tolerance zone of each dimension chain through unitized matching. And assemble the parts from sub-tolerance zone, which reduced the assembly tolerance range of dimension chains. This method can realize batch matching. Wang K. [12] takes the assembly accuracy and matching rate as evaluation indexes to establish a selective assembly model in the case of the multi- requirements. W.K put forward a improved Strength Pareto Evolutionary Algorithm 2 (SPEA2) for multi-objective complex mechanical products. By cutting the tolerance, this method decreases the selecting difficulty and enhances the matching rate. What's more, Chen [13] constructs an optimization model aiming at minimizing the fluctuation of assembly gap in unitized assembly by using multi-objective PSO which bring the sharing mechanism and dynamic archiving mechanism. The essence of unitized assembly is to realize the tolerance cutting, which considers both the assembly accuracy and matching rate. This method also has many disadvantages. For example, in order to guar- 
antee the matching rate, it will inevitably sacrifice the assembly accuracy in some degree. For general non-core components, the loss is controllable, but for the core components, the loss is not accepted. In addition, interference fit occurs many times in the process, especially in the long assembly sequences, and the interference fit will greatly increase the assembly cost.

Compared with the unitized assembly, the research on the precise assembly focus on Taguchi Method. P.F. [14] uses Taguchi quality model to measure the quality loss of each parameter in process. Aiming at the minimum process quality loss, A.Y [15] adopts the signal-to-noise ratio (SNR) to measure the influence weight of various indicators on the overall assembly quality fluctuation, and establishes a model based on the Atilesleon multivariate quality loss function.

To solve the above problems, this study proposes an improved Taguchi algorithm. The core components adopt the Target-is-best function, while the non-core components use the Improved-Smaller-is-better function, which could achieve convergence as soon as possible and increase the matching rate. The general parts adopt the ClassicalSmaller-is-better function, which can not only ensure the accuracy, but also reduce interference fit and assembly cost. The following chapters can be divided into two parts. The first part is the measurement of assembly precision loss, including the modelling hypothesis, objective function and matching model. The second part is a case study.

\section{The mathematic of the matching model for complex product assembly}

From the literatures, it is found that the assembly matching modes of different parts are different. For the noncore parts, the batch matching mode is adopted. For the core parts, the precise matching mode is selected. And the parts, which belongs neither non-cores nor cores, the unitized matching mode is the best choice.

Take the aircraft assembly process for example, the main parts of an aircraft include the cockpit, engine tower, forebay, central base, central cabin, horizontal and vertical tail fin and wing, etc., as shown in Fig. 1.

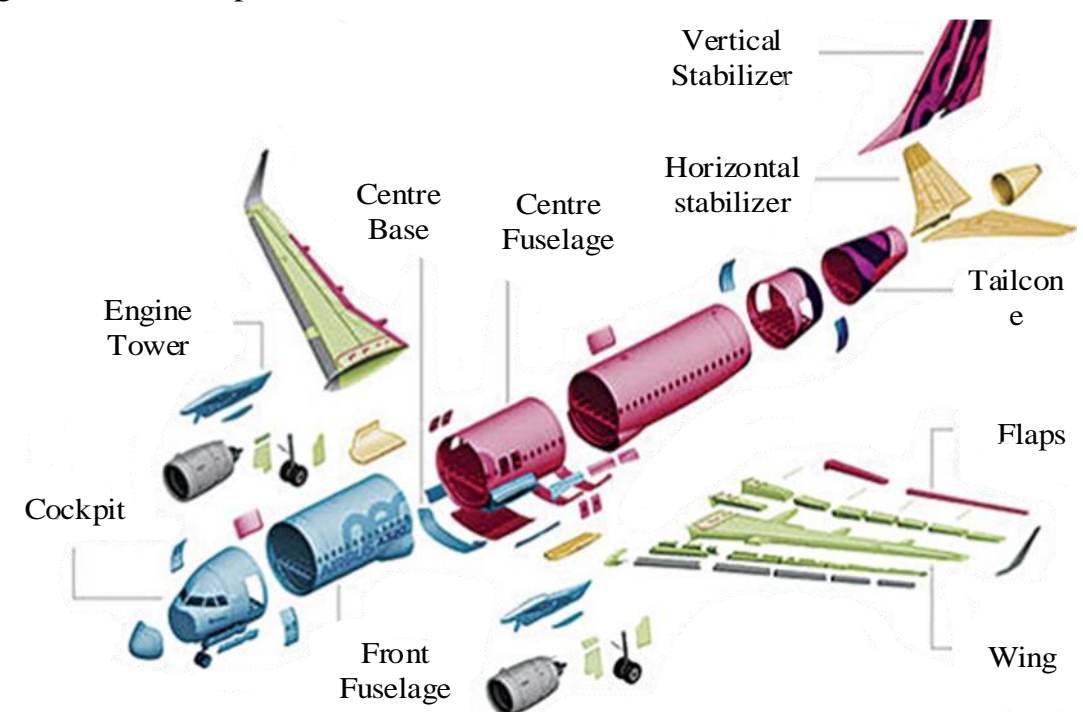

Fig. 1 The components of aircraft

The most important component is the engine. The main structure of the engine is shown in Fig. 2, including the transmission shaft, the first and second reducer, the power turbine, the compressor turbine, the compressor and the auxiliary trans mission at all levels.
According to the requirements of the importance and precision of each component, the structure importance level can be refined, as shown in Fig.3, where red represents the core components, blue represents the general components, and white represents the non-core components.

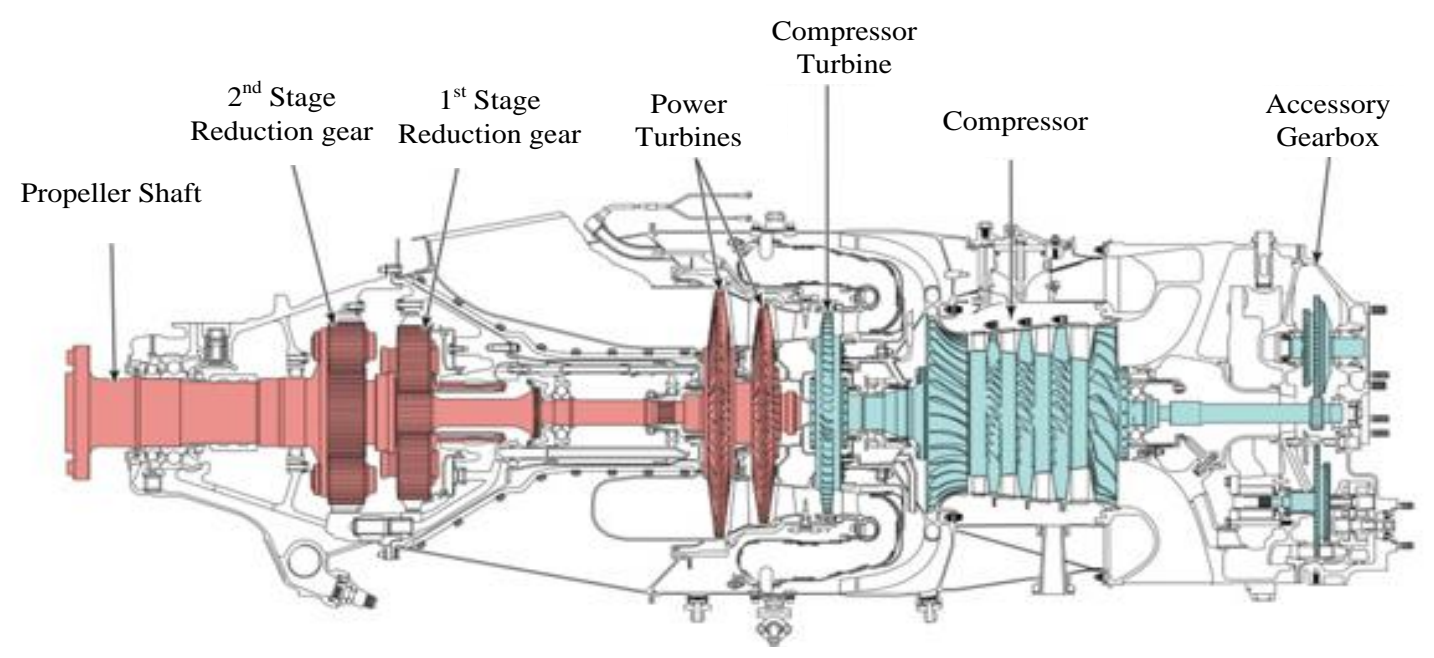

Fig. 2 The components of aircraft engine 


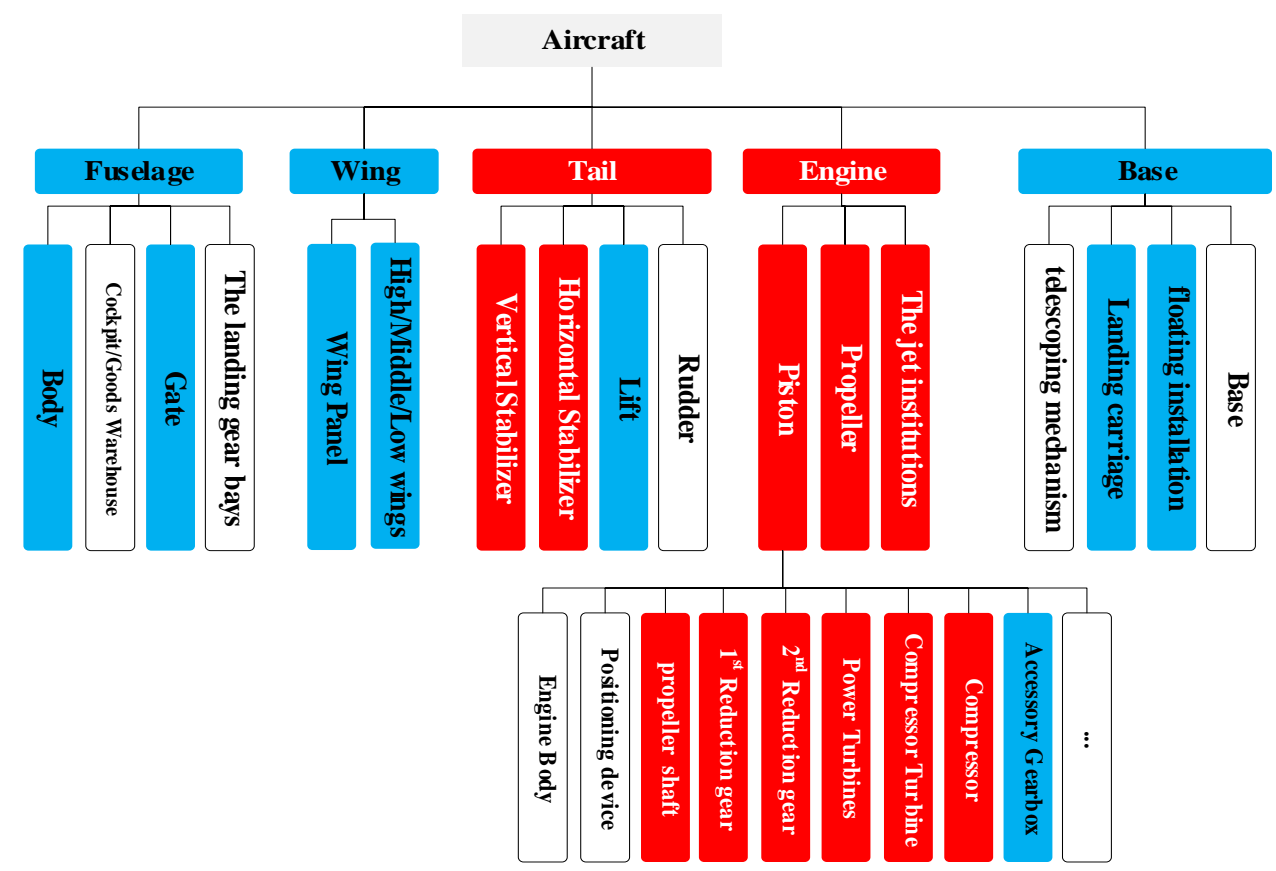

Fig. 3 The importance level of aircraft

According to different importance level, this study constructs the matching model. The Taguchi model is used to measure the deviation of dimension chains in the assembly process, and the genetic algorithm (GA) is used to solved the model. The following will be elaborated from three aspects of modeling hypothesis, Taguchi loss function (objective function) and the overall model.

\subsection{The modeling hypothesis of the matching}

In order to construct the matching model with both assembly accuracy and matching rate, different matching strategies are adopted for different important levels. As shown in Table 1.

Table 1

The modeling hypothesis of different components

\begin{tabular}{|c|c|c|c|}
\hline $\begin{array}{c}\text { Accu- } \\
\text { racy }\end{array}$ & $\begin{array}{c}\text { Match- } \\
\text { ing rate }\end{array}$ & Description & Modeling hypothesis \\
\hline Low & High & $\begin{array}{c}\text { Low cost; } \\
\text { Low Accuracy }\end{array}$ & $\begin{array}{c}\text { Improved-the- } \\
\text { Smaller-is-Better }\end{array}$ \\
\hline $\begin{array}{c}\text { Mid- } \\
\text { dle }\end{array}$ & Middle & $\begin{array}{c}\text { Not high cost; } \\
\text { Not low Accuracy }\end{array}$ & The-Smaller-is-Better \\
\hline High & Low & High Accuracy & The-Target-is-Best \\
\hline
\end{tabular}

In order to enhance the matching precision, the assembly gap should be close to 0 . While the interference fit whose assembly process need to be heated, will greatly increase the cost, in particular, the heating process requires repeated many times in complexity equipment. So this research put forward the matching model according to the different importance level and adopt different strategies. Such as the core component, for no caring the matching rate, need to be with high accuracy. So the matching strategy adopted the-Target-is-Best, whose assembly tolerance target is close to 0 . For general components, both cost and quality accuracy need to be taken into account, and the precision requirement is relatively high. Therefore, the measurement is carried out with the classical-the-Smaller-is-Better, which can improve the accuracy while reducing part of interference fit. The non-core components pay attention to the avoidable cost but it can accept low quality accuracy, so the assembly process adopt as many gap fit as possible in the assembly process. In order to reduce the interference, fit that may occur, this model adopt the improved-the-Smaller-is-Better. The specific model can be seen in the next section.

\subsection{Improved Taguchi loss function -objective function}

Taguchi quality loss function [16-17] is proposed to measure the assembly deviation. The model can be divided into the-Target-is-Best, the-Smaller-is-Better and theLarger-is-Better [18]. In the-Target-is-Best, the dimension chains $\mathrm{y}$ is expected to fluctuate around the target value $\mathrm{T}$, and the smaller the fluctuation is, the better the result is. In the-Smaller-is-Better, it is hoped that the closer the $\mathrm{y}$ is to the minimum value. This article does not cover the-Largeris-Better.

the-Target-is-Best is presented:

$$
L_{T}\left(y_{i}\right)=k\left(\frac{y_{i}-T_{i}}{T_{U}^{i}-T_{l}^{i}}\right)^{2} .
$$

The-Smaller-is-Better is:

$$
L_{L}\left(y_{i}\right)=k\left(\frac{y_{i}-T_{l}^{i}}{T_{U}^{i}-T_{l}^{i}}\right)^{2},
$$

where: $y_{i}$ is the measured dimension tolerance; $k$ is the average loss; $T_{U}^{i}, T_{l}^{i}$ is the lower and target values [19].

What's more, this study improves the-Smaller-isBetter. The $T_{l}$ is the target value set in the design period. This paper changes the $T_{i}$ into $T_{i}^{\prime}$. The $T_{i}^{\prime}$ is the dimension tolerance of preceding activity. This means that this study does not use the target value but the preceding activity value. By using the $T_{i}^{\prime}$.in the Smaller-is-Better, it can reduce the influence on the following activity and reduce the interference fit. This method also can be used in the green manufacturing and remanufacturing. The below equation is the 
improved-the-Smaller-is-Better[18].

$$
L_{L}\left(y_{i}\right)^{\prime}=k\left(\frac{y_{i}-T_{i}^{\prime}}{T_{U}^{i}-T_{l}^{i}}\right)^{2}
$$

where: $y_{i}$ is the measured dimension tolerance; $T_{i}^{\prime}$ is the dimension tolerance of preceding activity; $T_{i}^{\prime}=y_{n-1}$.

Dr. Taguchi proposed the method of multiple quality characteristics based on SNR [20] to unify the dimensions in the complex products:

SNR of the-Target-is-Best is:

$$
\eta_{T}=10 \lg \frac{\mu^{2}}{\sigma^{2}} \approx 10 \lg \frac{y^{-2}}{s^{2}} .
$$

The-Smaller-is-Better is:

$$
\eta_{S}=10 \lg \left(\frac{1}{\mu^{2}+\sigma^{2}}\right) \approx-10 \lg \left(\frac{1}{n} \sum_{i=1}^{n} y_{i}^{2}\right),
$$

where: $\eta_{T}$ and $\eta_{s}$ is the SNR. The SNR is the factor to show the deviation of tolerance loss. The bigger the SNR is, the less the loss is. And:

$$
\begin{aligned}
& \bar{y}=\frac{1}{n} \sum_{i=1}^{n} y_{i} \\
& s^{2}=\frac{1}{n-1} \sum_{i=1}^{n}(y-\bar{y})^{2}
\end{aligned} .
$$

SNR is adopted to represent the contribution. The equation of the weight is shown in (5) [18]:

$$
\lambda_{i}=\frac{1 / \eta_{i}}{\sum_{j=1}^{n} 1 / \eta_{j}} .
$$

This weight represents the same important level, and for different important levels, 5/3/1 is introduced.

\subsection{The architecture of the model}

This paper proposes a matching model on complex product by using improved Taguchi method. As shown in Fig.4, this model takes each dimension chain as a unit. If one component takes part in several dimension chains, all the dimension chains are taken in to compute. The SNR is the solution to handle the multi-dimensions in different dimension chains and different important level parts. And finally, the GA is introduced to get the matching solution.

The innovation of this method is mainly manifested as follows.

1) The dimension chain is the basic research object, rather than each assembly relation. This method can avoid the influence of preceding activity or following activity on subsequent process.

2) Assign the weights by SNR to distinguish the importance of components. Highlight the importance of core parts in matching, and avoid the problem that the noncore parts dimension chains are much more than the core dimension chains. This problem can lead the matching decision to be more based on the non-core components rather than the core ones.

3) The improve-the-Smaller-is-Better. This method improves the target value and are used in non-core assembly matching. It can ensure that the interference fit is as less as possible, and reduce the heating process, and cut down the avoidable cost.

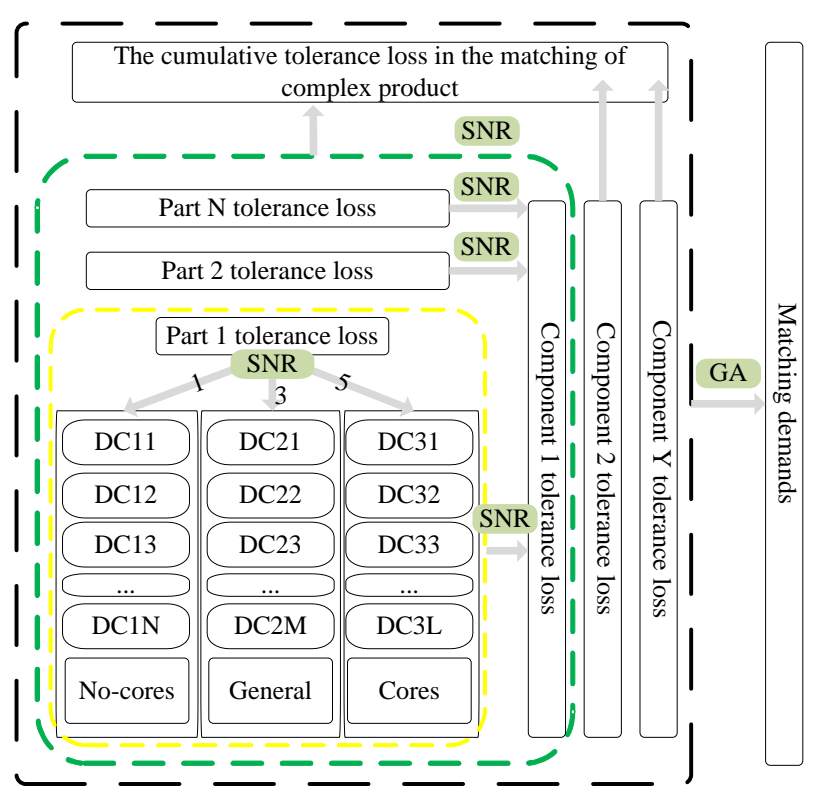

Fig. 4 The matching model based on improved Taguchi Method

\section{Case study}

Section 3 devoted to the basic aspects of the ALC. It is a decomposition-based and convergent collaborative optimization method [12]. Its primary thought is to allow each sub-decision-making-unit to make decisions independently and obtain global optimum through system partition and distributed decision-making system.

\subsection{The description of the model}

In order to test the effectiveness of the mathematical model, this study adopts a complex bolt assembly model as an example. From the perspective of the method mentioned in the Fig. 4, the complexity of the dimension chains in the testing model provides great insights about the positive correlation. 1) The core chains, the non-core chains and the general chains are regard as the primary limitation factors including in the test models. (2) And what's more, one part in several chains is another constraint. Arguing for the characteristic of the assembly model in Fig. 5 itself, the representative model covers all. Detailed discussed can be seen below the Table 2 .

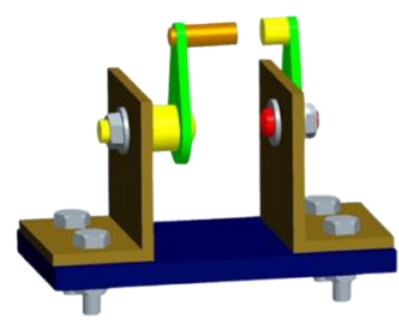

Fig. 5 The complex bolt assembly model 
According to the simulation example, a total of 12 parts are required for the assembly, show in Fig. 5. Dimension chain 1 is the core chain of the assembly and shown in Figs. 6-7.

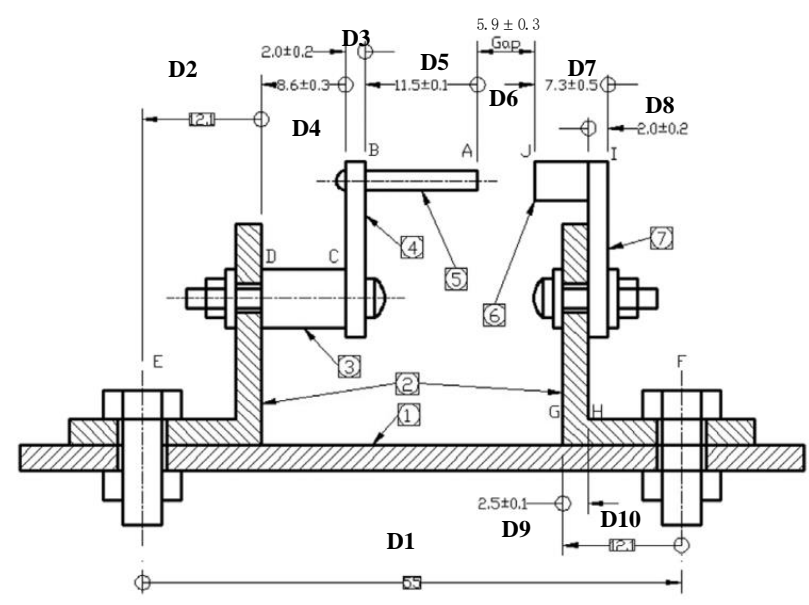

Fig. 6 Dimension chain 1 for complex bolt assembly

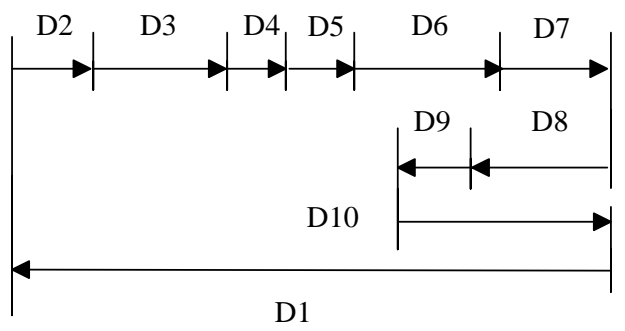

Fig. 7 Chain 1

The component 1 and 2 in Fig. 6 are fixed by four bolts on the base of the assembly. There are of four dimension chains, marked as chain 2 . They are non-cores, shown in Fig. 8.

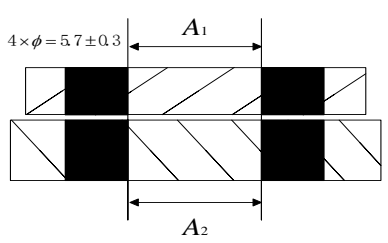

a

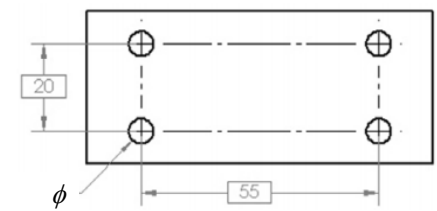

b
Fig. 8 Chain 2

In addition, the connection between the component 4 and 5 in Fig. 6 are hexagon connection, as shown in Fig. 9 , marked as chain 3 , general dimension chain.

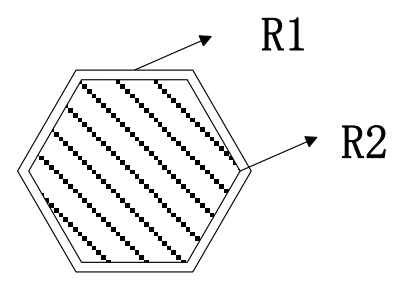

Fig. 9 Chain 3

As shown in Table 2, there are three types of chains in this product. Chain 1 is D1-D2-D3-D4-D5-D6-D7-D8D9-D10, which is the core chain of this assembly. Chain 2 is fixed through screw hole. There are 4 chains in the prod- uct and they are non-core dimension chains; Chain 3 is hexagonal dimension chain and general dimension chain. The assembly precision requirements are shown in Table 3. Among them, the situation that one part is in 2 chains exits. Such as part 2, it is in both chain 1 and two chains in chain 2 .

Table 2

The description of dimension chains

\begin{tabular}{|c|c|c|c|}
\hline D-Chains & Description & Symbol & $\begin{array}{c}\text { Tolerance, } \\
\mu \mathrm{m}\end{array}$ \\
\hline \multirow{10}{*}{1} & \multirow{10}{*}{$\begin{array}{l}\text { D1-D2-D3-D4-D5- } \\
\text { D6-D7-D8-D9-D10 }\end{array}$} & D1 & 0 \\
\hline & & D2 & 0 \\
\hline & & D3 & 0.3 \\
\hline & & D4 & 0.2 \\
\hline & & D5 & 0.1 \\
\hline & & D6 & 0.3 \\
\hline & & D7 & 0.5 \\
\hline & & D8 & 0.2 \\
\hline & & D9 & 0.1 \\
\hline & & D10 & 0 \\
\hline \multirow[b]{2}{*}{2} & \multirow{2}{*}{$\begin{array}{l}\Phi-A 1-\Phi-\Phi-A 2-\Phi \\
\Phi-A 3-\Phi-\Phi-A 4-\Phi \\
\Phi-A 5-\Phi-\Phi-A 6-\Phi \\
\Phi-A 7-\Phi-\Phi-A 8-\Phi\end{array}$} & $\Phi$ & 0.3 \\
\hline & & $\begin{array}{l}\mathrm{A} 1 \backslash \mathrm{A} 2 \backslash \mathrm{A} 3 \backslash \mathrm{A} 4 \\
\mathrm{~A} 5 \backslash \mathrm{A} 6 \backslash \mathrm{A} 7 \backslash \mathrm{A} 8\end{array}$ & 0.6 \\
\hline \multirow[b]{2}{*}{3} & \multirow{2}{*}{ R1-R2 } & R1 & 0.2 \\
\hline & & R2 & 0.1 \\
\hline
\end{tabular}

\subsection{Solving based on GA}

Encoding. The GA is the solution of this model. According to the situation of storage in factory, the parts serial number act as the encoding. The assembly contains a total of seven machined parts and five standard parts. The standard parts are not needed matching. In the remaining 7 parts, part 1 contains 9 dimensions, part 2 contains 5 dimensions, the inventory of parts is 100 . Binary encoding is chosen in the paper. Part 1 with 11 binaries, Part 2 with 10, the remaining are 7 binaries, shown in Fig. 10.

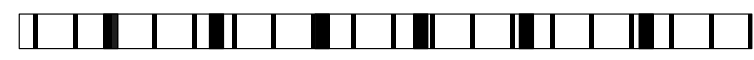 \\ Part $\square$}

Fig. 10 Encoding

In this study, fixed crossover probability is adopted, $\mathrm{Pc}=0.6$. In addition, Invert the gene values on one or some gene positions of individuals according to the fixed probability $\mathrm{Pm}=0.05$, and then the new individuals and mutation are achieved.

The Fitness function. By statistics, the mean and square root of each parameter in the dimension chain can be obtained.

Table 3

The parameters in Chain 1

\begin{tabular}{|c|c|c|c|c|}
\hline & $\sigma^{2}$ & $\mu$ & $\eta_{i}$ & $\lambda_{i}$ \\
\hline D3 & 0.021355 & 3.607108 & 27.84809 & 0.151897 \\
\hline D4 & 0.011423 & 2.018354 & 25.52233 & 0.165738 \\
\hline D5 & 0.00319 & 11.49667 & 46.17376 & 0.091611 \\
\hline D6 & 0.035493 & 5.933482 & 29.9648 & 0.141167 \\
\hline D7 & 0.095211 & 7.337592 & 27.52419 & 0.153684 \\
\hline D8 & 0.012707 & 1.978912 & 24.88825 & 0.169961 \\
\hline D9 & 0.002748 & 2.505162 & 33.58699 & 0.125942 \\
\hline
\end{tabular}

And the SNR and weight of each parameter can be 
calculated successively, as shown in Table 3. The D3 SNR is calculated in the below.

$$
\eta_{1}=10 \lg \frac{\mu^{2}}{\partial^{2}}=10 \times \lg \left(\frac{3.61^{2}}{0.0214}\right)=27.83 .
$$

The weight of D3 is:

$$
\lambda_{1}=\frac{\frac{1}{\eta_{1}}}{\sum_{j=1}^{n} \frac{1}{\eta_{j}}}=0.152
$$

Similarly, the weight of all the parameters can be obtained. There are 24 dimension chains needed to be assembled, and the fitness function can be obtained in Eq. (9).

The stopping criterion for iteration is genmax = 1000.

$$
\begin{aligned}
& L\left(y_{1}, y_{2}, \ldots, y_{11}\right)=\sum_{i=1}^{n} \lambda_{i} L\left(y_{i}\right)= \\
& =5\left(\lambda_{1} \frac{y_{1}-T_{1}^{\prime}}{T_{U}^{1}-T_{L}^{1}}+\ldots+\lambda_{7} \frac{y_{7}-T_{7}^{\prime}}{T_{U}^{7}-T_{L}^{7}}\right)+ \\
& +3\left(\lambda_{8} \frac{y_{8}-T_{8}^{\prime}}{T_{U}^{8}-T_{L}^{8}}+\ldots+\lambda_{23} \frac{y_{23}-T_{23}^{\prime}}{T_{U}^{23}-T_{L}^{23}}\right)+\lambda_{24} \frac{y_{24}-T_{24}{ }^{\prime 24}}{T_{U}^{24}-T_{L}^{24}} .
\end{aligned}
$$

\subsection{Result and discussion}

After the GA, the matching results obtained are shown in Table 4, with a total of 13 samples.
The horizontal axis represents the number of iterations, and the vertical axis represents the cumulative tolerance loss.

Table 4

Matching results

\begin{tabular}{|l|l|l|l|l|l|l|l|}
\hline No. & 1 & 2 & 3 & 4 & 5 & 6 & 7 \\
\hline 1 & 48 & 12 & 57 & 32 & 68 & 100 & 4 \\
\hline 2 & 11 & 12 & 89 & 74 & 31 & 63 & 69 \\
\hline 3 & 18 & 92 & 71 & 66 & 69 & 100 & 73 \\
\hline$\ldots$ & $\ldots$ & $\ldots$ & $\ldots$ & $\ldots$ & $\ldots$ & $\ldots$ & $\ldots$ \\
\hline
\end{tabular}

According the calculation, the cumulative loss fluctuates greatly at the beginning. The reasons can be expressed as follows. 1) the initial population is generated randomly, and the cross variation leads to large pulsation; 2) different levels of assembly losses lead to different contributions to the results, especially the assembly parameters of core parts will greatly affect the accumulation of assembly losses. As the number of iterations increases, the fluctuation tends to 1.76 , which indicates that when the number of iterations increases, the elite of all parameters will retain the selection results and gradually tend to be stable. When the number of iterations reaches 447 , it basically tends to be stable.

There are 4 comparative tests in this paper, as follows. And there are four indicators to evaluate the algorithm, 1 ) the convergence generation (represents the convergence rate); 2) the cumulative loss (represents the accuracy loss), 3 ) heating times (represents the number of interference fit, reflecting the avoidable cost); 4) matching result (represents the optimization result). A good algorithm should have faster convergence, less precision loss, fewer interference fit times and more optimal results. In another words, the smaller 1), 2) and 3) are, and the larger 4) are, the better the algorithm results will be.

Table 5

The compared test

\begin{tabular}{|l|l|l|l|l|l|}
\hline Item & Proposed Algorithm & Compared test 1 & Compared test 2 & Compared test 3 & Compared test 4 \\
\hline The convergence generation & 447 & 412 & 613 & 502 & None \\
\hline cumulative loss & 1.76 & 107.9 & 1.81 & 103.6 & 4829 \\
\hline Heating times & $2-4$ & $1-6$ & 5 & $3-6$ & 5 \\
\hline Matching result & 13 & 7 & 1 & 3 & None \\
\hline
\end{tabular}

Comparative test 1 adopts the unclassified method based on SNR, and all the other conditions are the same as Proposed Algorithm except the objective function.

$$
\begin{aligned}
& L\left(y_{1}, y_{2}, \ldots, y_{11}\right)=\sum_{i=1}^{n} \lambda_{i} L\left(y_{i}\right)= \\
& =5\left(\lambda_{1} \frac{y_{1}-T_{1}}{T_{U}^{1}-T_{L}^{1}}+\ldots+\lambda_{7} \frac{y_{7}-T_{7}}{T_{U}^{7}-T_{L}^{7}}\right)+ \\
& +3\left(\lambda_{8} \frac{y_{8}-T_{8}}{T_{U}^{8}-T_{L}^{8}}+\ldots+\lambda_{23} \frac{y_{23}-T_{23}}{T_{U}^{23}-T_{L}^{23}}\right)+\lambda_{24} \frac{y_{24}-T_{24}}{T_{U}^{24}-T_{L}^{24}} .
\end{aligned}
$$

Compared with the objective function of proposed Algorithm, the objective function of the comparative test 1 showed a trend of shrinking, with the dropping of fitness function, the quality loss present amplification, the frequency of heating gets higher and optimization results trends to less. So the proposed method is superior to the comparative test 1 on the matching precision, matching rate and heating times.

In comparative test 2 , the objective function construction method is based on the-Target-is-Best; in comparative test 3 , the objective function with the-Smaller-is-Better is adopted; in comparative test 4 , the random selection is adopted. According to Table 5, under the same conditions (probability of crossover mutation, initial population and termination conditions), the proposed algorithm has great advantages in convergence speed, heating assembly times and optimal results.

\section{Conclusion}

In this paper, the improved Taguchi methods for quality measures is used to calculate the tolerance loss of dimension chains. The important degree and SNR are introduced to measure the different chains to avoid the non-core influence. The model acts as the fitness function of genetic algorithm, in meet the precision request of complex prod- 
ucts matching. This model pays more attention to the accuracy of core parts and assembly cost of non-core parts. In order to ensure the accuracy in the non-cores, the effect of preceding activity and following activity can be reduced by improved-the-Smaller-is-Better. Since this model can handle the preceding activity assembly, it can also be used in the green manufacturing and remanufacturing.

Through case analysis, it is found that the assembly matching has great advantages in convergence speed, matching rate and assembly cost. But the study found that when the parts in multiple non-core dimension chains, especially the chains are quite large, the model works failure. Therefore, one of the emphases on the further research is to optimize multiple dimension chains in the precision on huge non-core components.

\section{Acknowledgments}

This work was financially supported by the Fundamental Research Funds for the Central Universities (No. B200201054).

\section{References}

1. Trovati, M.; Zhang, H.; Ray J.; Xu, X. 2020. An entropy-based approach to real-time information extraction for industry 4.0, IEEE Transactions on Industrial Informatics 16(9): 6033-6041. https://dx.doi.org/10.1109/TII.2019.2962029. .

2. Björn, S. 2016. Futuring european industry-assessing the manufuture road towards EU reindustrialization, European Journal of Futures Research 4(1): 25-36. https://dx.doi.org/10.1007/s40309-016-0100-6.

3. Davis, J.; Edgar, T.; Porter, J. 2012. Smart manufacturing, manufacturing intelligence and demand-dynamic performance, Computers \& Chemical Engineering 47(1), $145-156$. https://dx.doi.org/10.1016/j.compchemeng.2012.06.037.

4. Kamble, S.; Gunasekaran, A.; Ghadge, D. A. 2020. A performance measurement system for industry 4.0 enabled smart manufacturing system in SMMEs: a review and empirical investigation, International Journal of Production Economics 229:107853.

https://dx.doi.org/10.1016/j.ijpe.2020.107853.

5. Li, L.; Yong, W.; Kuo, L. 2021. Preventive maintenance scheduling optimization based on opportunistic production-maintenance synchronization. Journal of Intelligent Manufacturing 32(1): 545-558. https://doi.org/10.1007/s10845-020-01588-9.

6. Park, J. 2015. Technology and issue on embodiment of smart factory in smaller-medium manufacturing business, The Journal of Korean Institute of Communications and Information Sciences 40(12): 2491-2502. https://dx.doi.org/10.7840/kics.2015.40.12.2491.

7. Banks M. 2018. UK catapults fall short, claims review, Physics World 31(1): 10. https://dx.doi.org/10.1088/2058-7058/31/1/15.

8. Wang X.; Liu M.; Ge M.; MA J.; Liu C. 2016. Online control threshold optimization for complex mechanical products assembly process based on hybrid genetic particle swarm optimization, Journal of Mechanical Engineering 52(1): 130-138.
https://dx.doi.org/10.3901/JME.2016.01.130.

9. Lu, C.; Zhang, F.; Wang ,H.; Yu, Y. 2016. Assembly quality analysis of the front face of the tiny electric car based on the virtual assembly, Machinery Design \& Manufacture 1(7): 232-235.

https://dx.doi.org/10.19356/j.cnki.10013997.2016.07.060.

10. Sun, Y.; Ni, Y.; Pan, Q. 2016. Assembly accuracy analysis and prediction of a four axis precision machine table, Mechanical Science and Technology for Aerospace Engineering 35(1): 44-49.

https://dx.doi.org/10.13433/j.cnki.10038728.2016.0108.

11. Wang, W. M.; Li, D. B.; He, F. 2018. Modelling and optimization for a selective assembly process of parts with non-normal distribution, International Journal of Simulation Modelling 17(1): 133-146. https://dx.doi.org/ 10.2507/IJSIMM17(1)CO1.

12. Wang, K.; Zhang, S.; He, W. 2016. Selective assembly of complicated mechanical product based on SPEA2, Journal of Shanghai Jiaotong University 50(7): 1047-1053. https://dx.doi.org/10.16183/j.cnki.jsjtu.2016.07.011.

13. Chen, J.; Xu, S. 2013. Optimization of selective assembly with multiple quality characteristics based on MOPSO, China Mechanical Engineering 18: 24422447.

https://dx.doi.org/10.3969/j.issn.1004132X.2013.18.006.

14. Pei, F.; Tong, Y.; Li, D. 2018. Multi-level welding quality fault discovery of an intelligent production line by using taguchi quality loss function and signal-noise ratio, IEEE Access 6: 40792-40803.

https://dx.doi.org/10.1109/ACCESS.2018.2857506.

15. Ai, Y.; Tian, J.; Gao, H. 2012. Study on assembly quality optimization techniques based on multivariate quality loss and genetic algorithm, China Mechanical Engineering 23(10): 1161-1164.

https://dx.doi.org/0.3969/j.issn.1004-132X.2012.10.008.

16. Pedersen, S. N.; Howard, T. 2016. Data acquisition for quality loss function modelling, Procedia Cirp 43(1), 112-117. https://dx.doi.org/10.1016/j.procir.2016.02.032.

17. Antony, J.; Perry, D.; Wang, C.; Kumar, M. 2006. An application of Taguchi method of experimental design for new product design and development process. Assembly Automation 26(1):18-24.. https://dx.doi.org/10.1108/01445150610645611.

18. Yoon, Y. J.; Kim, H.; Yoo, J. 2017. Modified loss function for the quality management in service industry, Advanced Science Letters 23(10): 9403-9406. https://dx.doi.org/10.1166/asl.2017.9712.

19. Delgoshaei, A.; Ariffin, A.; Ali, A. 2018. A Multi-period scheduling method for trading-off between skilledworkers allocation and outsource service usage in dynamic cms, International Journal of Production Research 55(4): 997-1039. https://dx.doi.org/10.1080/00207543.2016.1213445.

20. Taguchi, G. 1995. Quality engineering (Taguchi methods) for the development of electronic circuit technology, IEEE Transactions on Reliability 44(2):225229. https://dx.doi.org/ 10.1109/24.387375. 
F. Pei, Y. Tong, M. Yuan, H. Song

\section{RESEARCH ON COMPLEX PRODUCT PARTS MATCHING BY USING IMPROVED TAGUCHI METHOD}

\section{S u m m a r y}

With the development of intelligent manufacturing, the key strategic of complex equipment is becoming more and more obvious. How to realize the assembly of complex products has become the focus of intelligent manufacturing. This paper puts forward the improved Taguchi method to dimension chains measures, by using different quality loss function to different dimension chains, the cores are the Target-is-best, non-core is measured with the improved Smaller-is-better to improve convergence perusal and increase matching rate; General adopt Smaller-is-better to enhance assembly accuracy, reduce interference fit and assembly cost. Then the dimension chains quantitative model of complicated product assembly by using the signalto-noise ratio and different weights is built up. The model contains modeling assumption, the objective function and the matching model. And this model is regard as the fitness function of genetic algorithm. Finally, the feasibility and efficiency of the scheme are verified by the case study.

Keywords: Taguchi methods; dimension chains; GA; improved smaller-is-better method.

Received December 21, 2020 Accepted October 04, 2021

This article is an Open Access article distributed under the terms and conditions of the Creative Commons Attribution 4.0 (CC BY 4.0) License (http://creativecommons.org/licenses/by/4.0/). 